\title{
BMJ Open Multicentre prospective observational study protocol for radiation exposure from gastrointestinal fluoroscopic procedures (REX-GI study)
}

\begin{abstract}
Tsutomu Nishida (D) , ${ }^{1}$ Shiro Hayashi, ${ }^{1,2}$ Mamoru Takenaka, ${ }^{3}$ Makoto Hosono, ${ }^{4}$ Hirofumi Kogure, ${ }^{5}$ Kenkei Hasatani, ${ }^{6}$ Shinjiro Yamaguchi, ${ }^{7}$ Hirotsugu Maruyama, ${ }^{8}$ Hisashi Doyama, ${ }^{9}$ Hideyuki Ihara, ${ }^{10}$ Toshiyuki Yoshio, ${ }^{11}$ Koji Nagaike, ${ }^{12}$ Takuya Yamada, ${ }^{13}$ Takayuki Yakushijin, ${ }^{14}$ Tadayuki Takagi, ${ }^{15}$ Hidetaka Tsumura, ${ }^{16}$ Akira Kurita, ${ }^{17}$ Satoshi Asai, ${ }^{18}$ Yukiko Ito, ${ }^{19}$ Toshio Kuwai, ${ }^{20}$ Yasuki Hori, ${ }^{21}$ Iruru Maetani, ${ }^{22}$ Kenji Ikezawa, ${ }^{23}$ Takuji Iwashita, ${ }^{24}$ Kengo Matsumoto, ${ }^{1}$ Masami Inada, ${ }^{1}$ On behalf of the FIGHT Japan Group
\end{abstract}

To cite: Nishida T, Hayashi S, Takenaka M, et al. Multicentre prospective observational study protocol for radiation exposure from gastrointestinal fluoroscopic procedures (REX-Gl study). BMJ Open 2020;10:e033604. doi:10.1136/ bmjopen-2019-033604

- Prepublication history for this paper is available online. To view these files, please visit the journal online (http://dx.doi org/10.1136/bmjopen-2019033604).

Received 13 August 2019 Revised 20 December 2019 Accepted 20 December 2019

Check for updates

(C) Author(s) (or their employer(s)) 2020. Re-use permitted under CC BY-NC. No commercial re-use. See rights and permissions. Published by BMJ.

For numbered affiliations see end of article.

Correspondence to Dr Tsutomu Nishida; tnishida.gastro@gmail.com

\section{ABSTRACT}

Introduction Recently, the use of various endoscopic procedures under $\mathrm{X}$-ray fluoroscopic guidance, such as endoscopic retrograde cholangiopancreatography (ERCP), interventional endoscopic ultrasonography (EUS), enteral endoscopy and stenting, has been rapidly increasing because of the minimally invasive nature of these procedures compared with that of surgical intervention. With the spread of CT and fluoroscopic interventions, including endoscopic procedures under X-ray guidance, high levels of radiation exposure (RE) from medical imaging have led to major concerns throughout society. However, information about RE related to these imageguided procedures in gastrointestinal endoscopy is scarce, and the RE reference levels have not been established. The aim of this study is to prospectively collect the actual $\mathrm{RE}$ dose and to help establish diagnostic reference levels (DRLs) in the field of gastroenterology in Japan.

Methods and analysis This is a multicentre, prospective observational study that is being conducted to collect the actual RE from treatments and diagnostic procedures, including ERCP, interventional EUS, balloonassisted enteroscopy, enteral metallic stent placement and enteral tube placement. We will measure the total fluoroscopy time (min), the total dose-area product $\left(\mathrm{Gycm}^{2}\right)$ and air-kerma (mGy) of those procedures. Because we are collecting the actual RE data and identifying the influential factors through a prospective, nationwide design, this study will provide guidance regarding the DRLs of ERCP, interventional EUS, balloonassisted enteroscopy, enteral metallic stent placement and enteral tube placement.

Ethics and dissemination Approval was obtained from the Institutional Review Board of Toyonaka Municipal Hospital (25 April 2019). The need for informed consent will be waived via the opt-out method of each hospital website.

Trial registration number The UMIN Clinical Trials Registry, UMIN000036525.
Strengths and limitations of this study

The large, multicentre, nationwide dataset of radiation exposure doses for gastrointestinal fluoroscopic procedures in gastrointestinal endoscopy gathered in this study will serve as a basis for the development of diagnostic reference levels (DRLS) in Japan.

- Gastrointestinal fluoroscopic procedures have been rapidly increasing in number and complexity, but there are still not enough available local and national DRLs in gastrointestinal endoscopy units.

- These data may not be valid for old models of fluoroscopic systems because this study will include data from fluoroscopic systems with available radiation data.

\section{INTRODUCTION}

Medical radiation is widely used in both medical imaging and radiation treatment. In medical imaging, fluoroscopy employs radiation to show a continuous X-ray image on a monitor and plays a major role in the daily practices of gastroenterology, digestive endoscopy, and hepatobiliary and pancreatic studies. Radiological medical imaging has both benefits and drawbacks for patients. The latter is split into two types: deterministic risks, ${ }^{1}$ determined by the threshold dose, as represented by skin injury and stochastic risks, determined by a linear no-threshold model, such as the cancer risk. ${ }^{2}$ There have been some reports on radiation-induced skin injury in cardiology and interventional radiology (IVR), ${ }^{3}$ but reports from gastrointestinal endoscopy units are rare. However, all medical staff in gastrointestinal endoscopy units need to have correct knowledge of the 
appropriate use of medical radiation. Historically, the use of medical radiation has rapidly increased since the 1990s with the spread of CT, and the radiation-associated cancer risk was recognised in the same period, even when the doses of radiation were small. ${ }^{4-6}$ In particular, the use of CT has increased approximately 12-fold in the UK and more than 20 -fold in the USA in the last 25 years. ${ }^{7}$

The International Atomic Energy Agency, the International Commission on Radiological Protection (ICRP), the United Nations Scientific Committee on the Effects of Atomic Radiation and other radiological societies have been attempting to manage medical radiation exposure (RE) according to the 'as low as reasonably achievable' principle by establishing diagnostic reference levels (DRLs) to optimise protection from medical radiation. The concept of DRLs was first introduced by the ICRP $73^{8}$ in 1996. Then, the ICRP emphasised the important role of DRLs as a tool for optimising patient protection. ${ }^{9} 10$ Accordingly, the ICRP sets specific target levels for various X-ray-related procedures in 2007. ${ }^{9}$ This movement of setting DRLs has been led by radiation-related societies in each region, although the movement has mainly been driven by Western countries. The ICRP 135 recommends that all individuals who are involved in patient procedures with the risk of medical exposure should be familiar with the DRL process as a tool for optimising protection. ${ }^{11}$ DRLs are now widely accepted in not only Western countries but also in Japan (Japan DRLs 2015), ${ }^{12}$ and DRLs have become the global standard for all procedures that use ionising radiation. Legislation has made it mandatory to establish and record DRLs in Europe, but that is not the case worldwide. The introduction of DRLs in the UK achieved a reduction of approximately $50 \%$ in the radiation dose in typical X-ray examinations over 15 years. ${ }^{13}$ However, there is still not enough available data on RE for gastrointestinal fluoroscopic procedures, such as endoscopic retrograde cholangiopancreatography (ERCP), interventional endoscopic ultrasonography (EUS), small bowel endoscopy and enteral stent placement; these techniques are still being developed and have recently been used with increasing frequency. ${ }^{1415}$

Our gastroenterologists and endoscopists are still unfamiliar with the DRL concept. Among the guidelines developed by gastrointestinal endoscopy associations, the 2012 European Society of Gastrointestinal Endoscopy guidelines for radiation protection state that the entrance skin dose (approximately equivalent to air-kerma (AK) in this study) and kerma-area product (KAP; approximately equivalent to the dose-area product (DAP) in this study) during diagnostic and therapeutic ERCP are 55-347 mGy and 3-115/8-333 $\mathrm{Gycm}^{2}$, respectively, although information regarding the DRLs of ERCP is limited because this statement is based on only approximately 600 cases of ERCP in 7 reports. ${ }^{14}$ No guidelines on RE from the American Society for Gastrointestinal Endoscopy (ASGE) exist, but the ASGE recommends measuring and documenting fluoroscopy time (FT) and radiation dose in all ERCP procedures as a quality indicator (level of evidence:
2C). ${ }^{16}$ Although no guidelines for exposure have been developed by the Japan Gastroenterological Endoscopy Society, a description of FT exists in the item regarding ERCP in the Japan Endoscopy Database, ${ }^{17}$ which is scheduled to be implemented as a nationwide endoscopic survey in 2020.

Recently, various endoscopic procedures performed under fluoroscopic guidance are rapidly increasing in popularity in gastrointestinal endoscopy units, where the aim is not only diagnosis but also therapeutic intervention. The ICRP recommends that DRLs should be used to manage patient doses during both diagnostic and interventional procedures. There is difficulty in applying the DRL concept to interventional procedures because the RE level depends on the complexity of the procedure and the individual clinical circumstances. ${ }^{10} 1819$ There have been attempts to establish DRLs for IVR procedures, where grouping by disease site may help minimise the wide distribution of RE. ${ }^{20} 21$

The Japanese DRLs were established on a basis of a survey and released in 2015; these guidelines defined the DRL value for fluoroscopically guided interventional procedures as a fluoroscopic radiation dose rate (RDR; interventional reference point dose rate) of $20 \mathrm{mGy} /$ min. ${ }^{12}$ However, it did not include information for specific procedures in the field of gastroenterology. ${ }^{12}$ Therefore, we aim to prospectively collect actual RE data and identify the influential factors, such as disease site, in the REX-GI (radiation exposure from gastrointestinal fluoroscopic procedures) study and to establish DRLs for the following interventional procedures in gastrointestinal endoscopy units: ERCP, interventional EUS, balloon-assisted enteroscopy, enteral metallic stent placement and enteral tube placement.

\section{METHODS AND ANALYSIS \\ Aims}

The primary aim of this nationwide, prospective study is to collect actual data on RE and identify the factors affecting $\mathrm{RE}$ during treatments and diagnostic procedures under different types of fluoroscopic guidance for gastroenterology procedures, including the gastrointestinal, hepatobiliary and pancreatic fields, to serve as a basis for the establishment of DRLs in Japan.

\section{Design}

This is a multicentre, prospective observational cohort study of consecutive patients undergoing the following five treatments and diagnostic procedures under fluoroscopic guidance in the field of gastroenterology: (1) ERCP, (2) interventional EUS, (3) balloon-assisted enteroscopy, (4) enteral metallic stent placement and (5) enteral tube placement. We will examine the procedure time ( $\mathrm{min})$, total FT (min), AK (mGy), DAP $\left(\mathrm{Gycm}^{2}\right),{ }^{2}$ total number of roentgenography procedures and $\mathrm{RDR}(\mathrm{mGy} / \mathrm{min})$ during the procedures. The participating clinicians will manage patients according to the usual clinical practice, 
Table 1 Fluoroscopic system and units performing procedures under fluoroscopic guidance

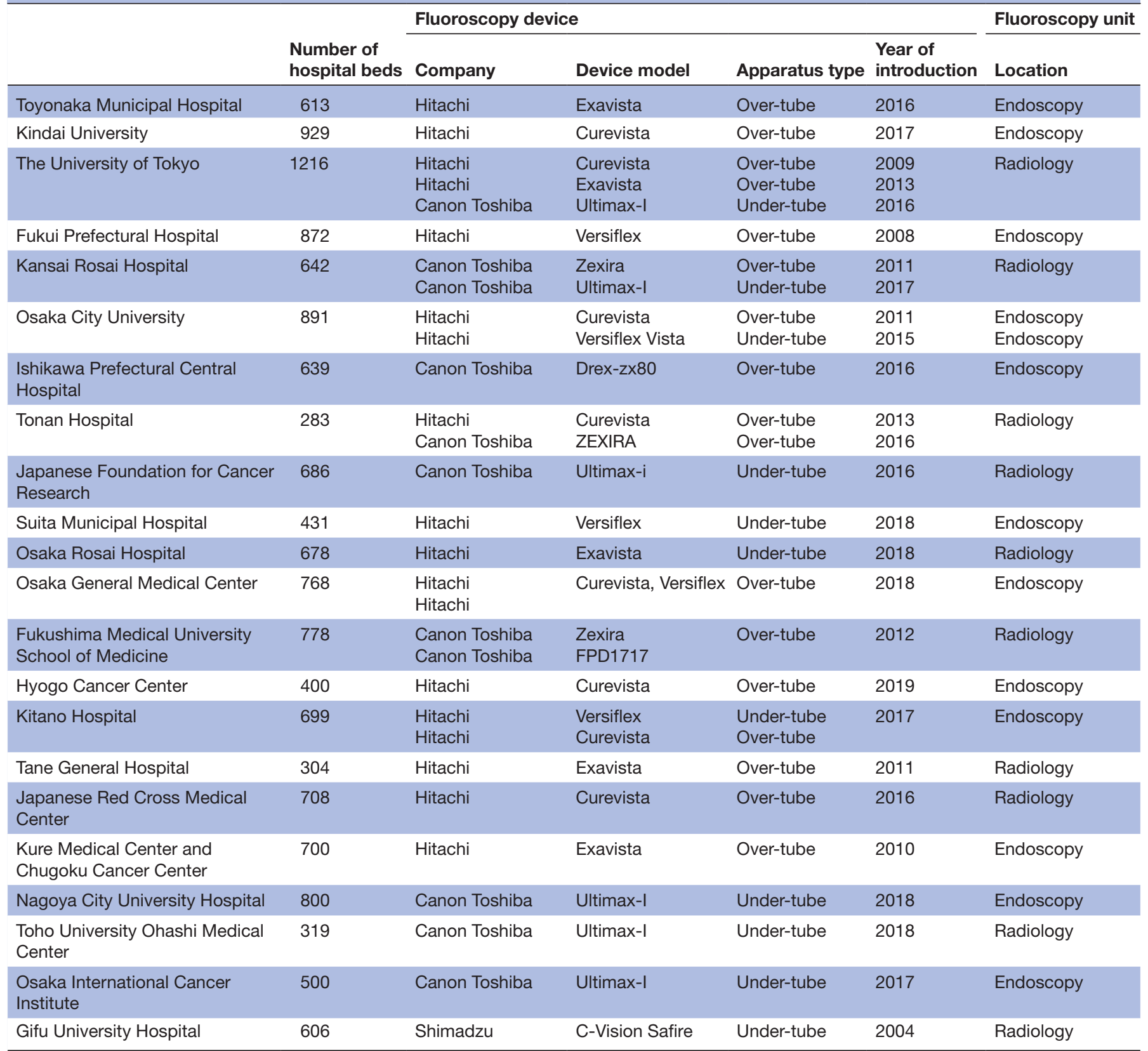

and the patients will undergo the above five procedures. For the analysis, all data, including the related variables and outcome data (tables 1 and 2), will be collected for all patients. The REX-GI study was registered with the UMIN Clinical Trials Registry at http://www.umin.ac.jp/ ctr/ under the number UMIN000036525 (registered 1 May 2019).

\section{Setting}

This study will be conducted at seven university hospitals, four cancer centres, nine general hospitals and two municipal hospitals in Japan. The participating hospitals are Toyonaka Municipal Hospital, Kindai University, the University of Tokyo, Fukui Prefectural Hospital, Kansai
Rosai Hospital, Osaka City University, Ishikawa Prefectural Central Hospital, Tonan Hospital, Japanese Foundation for Cancer Research, Suita Municipal Hospital, Osaka Rosai Hospital, Osaka General Medical Center, Fukushima Medical University School of Medicine, Hyogo Cancer Center, Kitano Hospital, Tane General Hospital, Japanese Red Cross Medical Center, Kure Medical Center and Chugoku Cancer Center, Nagoya City University Hospital, Toho University Ohashi Medical Center, Osaka International Cancer Institute and Gifu University Hospital (figure 1). Table 1 lists the fluoroscopic systems and units performing procedures under fluoroscopic guidance in each institution. The central sites of 


\begin{tabular}{ll} 
Table 2 & Primary outcomes \\
\hline Factors & Variables \\
\hline Patients $^{*}$ & Procedure type \\
& Age \\
& Sex \\
Fluoroscopic & Fluoroscopic device (company, device \\
system & model and manufacturing year) \\
& Basic use setting: frame per second and \\
& radiation field (cm ${ }^{2}$ † \\
Radiation & Total fluoroscopy time (min) \\
exposure & Air-Kerma (mGy) \\
& Dose-area product (Gycm $\left.{ }^{2}\right)$ \\
& Total number of roentgenography \\
& procedures \\
& Radiation dose rate (mGy/min)
\end{tabular}

*We will not collect patient weight or height because we have selected patients of standard size for the Japanese population, whose weight will range from 50 to $70 \mathrm{~kg}$.

tWhen the setting changes during the procedure, we will record the basic setting.

this study are located at the Toyonaka Municipal Hospital and Kindai University. The participating physicians are gastroenterologists or endoscopists, including all experts and trainees working at all involved hospitals. The quality of the fluoroscopic devices will be regularly monitored according to the procedures in each institution.

\section{Study population}

We will include all patients receiving usual clinical care who undergo the following treatments and diagnostic procedures under fluoroscopic guidance: (1) ERCP; (2) interventional EUS; (3) balloon-assisted enteroscopy; (4) enteral metallic stent placement and (5) enteral tube placement. There is no age restriction. We will exclude patients who do not want to participate in this study via the opt-out method on each hospital website and patients who the attending physicians judge to be unsuitable for inclusion in this study.

\section{Primary outcomes}

The primary outcomes will be the total FT (min), RDR $(\mathrm{mGy} / \mathrm{min})$, dose-area parameters (AK $(\mathrm{mGy})$ and DAP $\left.\left(\mathrm{Gycm}^{2}\right)\right)$ and the total number of imaging studies that the patients who meet the individual inclusion and exclusion criteria will undergo (table 2).

\section{Secondary outcome}

The secondary outcome will be the RE-related factors that affect the radiation dose in each procedure. The details are given in table 3 .

\section{Setting the sample size}

According to the preliminary questionnaire survey (data not shown), the numbers of examinations per year in the 8 centres that plan to participate in March 2019 are as follows: 4000 ERCP procedures, 125 EUS procedures, 320 small intestine endoscopy procedures, 44 esophageal stent placements, 150 gastroduodenal stent placements, 75 colorectal stent placements, 180 transanal ileus tube placements and 75 ileus tube placements. The ICRP 135 recommends using data from 20 to 30 facilities to set national DRLs, and a survey for a particular examination in a facility should usually involve the collection of data from at least 20 patients. ${ }^{11}$

To set the DRLs and to reduce intraprocedural variability in each hospital, we set the minimum sample size to at least 400 patients for each procedure. We believe that initially enrolling a high number of facilities and patients is desirable; therefore, we did not set an upper limit for the goals.
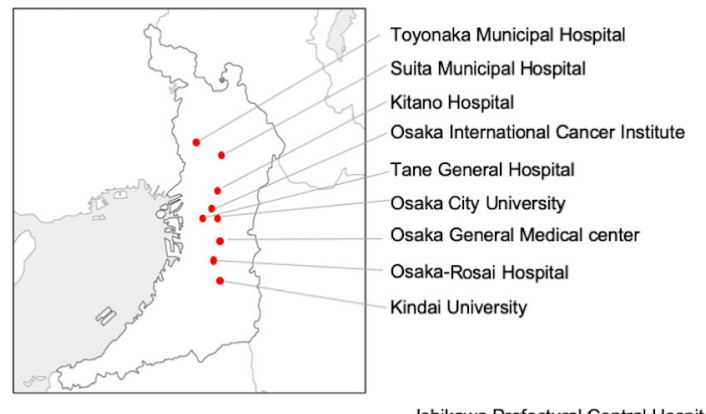

Ishikawa Prefectural Central Hospital
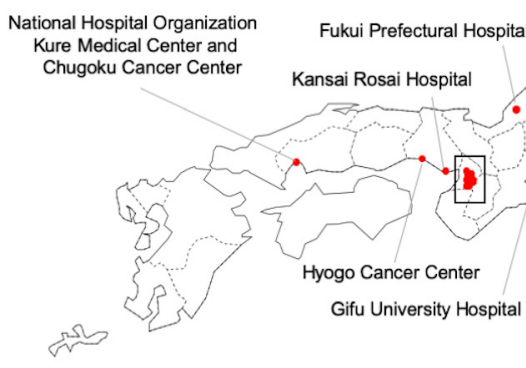

Gifu University Hospital

Giv University Hospital

Figure 1 The participating hospitals in this study. 
Table 3 Secondary outcomes

\section{Procedures Radiation exposure-related factors}

ERCP

1. Surgically altered gastrointestinal anatomy.

Billroth I reconstruction, Billroth II reconstruction, Roux-en-Y reconstruction and pancreaticoduodenectomy

2. Type of endoscope.

3. Naïve papilla.

4. Indications for ERCP (including suspicion) are classified into the following five categories:

a. Choledocholithiasis (maximum diameter, number of stones, presence of cholangitis, tube exchange for the above diseases, treatment for choledocholithiasis with or without balloon catheter, basket catheter, crusher, etc).

b. Distant malignant bile duct stricture (papillary tumour, distal cholangiocarcinoma, pancreatic cancer, etc).

c. Proximal malignant bile duct stricture (Hilar cholangiocarcinoma, intrahepatic cholangiocarcinoma, gallbladder cancer, etc).

d. Pancreatic duct examination (pancreas cancer, intraductal papillary mucinous neoplasm, etc).

e. Other diseases apart from those listed above (benign bile duct stricture, pancreatobiliary junction abnormality, etc).

5. Total procedure time (min).*

a. Cannulation time.

b. Treatment time.

6. Experience of the HVE or LVE. $\dagger$

7. Facility scale: the number of ERCP procedures per year.

8. Whether the fluoroscopic operator is inside or outside in the fluoroscopy room.

9. Various treatments (endoscopic sphincterotomy, stone treatment, bile duct/pancreatic stent, cytology, biopsy, naïve papilla, cannulation method, contrast agent, intubation time, first-use catheter, large balloon, crusher, drainage area or method, stent type used and cholangioscopy).

10. Sedation: medication and the depth of the anaesthesia. $\neq$

Interventional EUS

1. Indication for interventional EUS (EUS-guided hepaticogastrostomy), choledochoduodenostomy, cyst drainage, antegrade treatment, rendezvous technique and pancreatic duct drainage.

2. Total procedure time. $\neq$

a. Endoscope insertion time.

b. Treatment time.

3. Facility scale: the number of EUS interventions per year and the number of EUS-guided fine-needle aspiration procedures per year.

4. Double stenting (presence or absence of duodenal stenosis).

5. Device.

6. Scope position.

7. Sedation: medication and the depth of anaesthesia.

Balloon-assisted enteroscopy
1. Disease indicating balloon-assisted enteroscopy.

a. Hemostatic or bleeding confirmation.

b. Crohn's disease.

c. Small intestine tumour examination.

d. Others.

2. Insertion site: perioral or transanal.

3. Insertion length $(\mathrm{cm})$.

4. Total procedure time ( $\mathrm{min})$.

1. Stent location.

Enteral metallic stent placement
a. Oesophagus (upper/mid-low/trans).
b. Gastro-duodenum (above pylorus/trans pylorus/below pylorus).
c. Colon stent (right/left/rectum).

2. Total procedure time $(\min ) . \S$

a. Endoscope insertion time.

b. Treatment time.

1. Disease indicating ileus tube.

2. Intranasal ileus tube insertion for ileal obstruction or transanal ileus tube insertion for malignant colonic obstruction.

a. Tube insertion length for peroral ileus tube placement $(\mathrm{cm})$.

b. The occlusion site for the transanal tube (right/left/rectum).

3. Total procedure time (min). I 
Table 3 Continued

Procedures Radiation exposure-related factors

${ }^{*}$ Cannulation time is defined as the time from endoscope insertion until successful biliary cannulation, and treatment time is defined as the time from successful biliary cannulation until the scope is removed from the patient. The total procedure time is defined as the time from endoscope insertion until the scope is removed from the patient (cannulation time+treatment time).

THVE: endoscopists with more than 200 ERCP results and who have been involved in ERCP for over 10years. LVE: non-HVE endoscopists who perform ERCP.

‡Depth of anaesthesia is divided into three levels based on the RASS, Ramsay Scale and SAS: good, poor and very bad. The good level is defined as RASS score: -5--1, SAS score: 1-3 and Ramsay score: 3-6 equivalent, without additional unplanned doses. The poor level is defined as RASS score: $0-+1$, SAS score: $4-5$ and Ramsay score: $1-2$, without physical restraint but with unplanned doses. The very bad level is defined as requiring physical restraint with a force considered dangerous, RASS score: $+2-+4$, and SAS score: $6-7$ regardless of Ramsay score.

$\S$ Endoscope insertion time is defined as the time from endoscope insertion until the initial EUS-guided needle puncture, and treatment time is defined as the time from initial EUS-guided needle puncture until the scope is removed from the patient. The total procedure time is defined as the time from endoscope insertion until the scope is removed from the patient (endoscope insertion time + treatment time). ףEndoscope insertion time is defined as the time from endoscope insertion until initial guidewire exploration, and treatment time is defined as the time from initial guidewire exploration until the scope is removed from the patient. The total procedure time is defined as the time from endoscope insertion until the scope is removed from the patient (endoscope insertion time + treatment time).

ERCP, endoscopic retrograde cholangiopancreatography; EUS, endoscopic ultrasonography; HVE, high-volume endoscopist; LVE, lowvolume endoscopist; RASS, Richmond Agitation-Sedation Scale; SAS, Sedation-Agitation Scale.

\section{Data analysis plan}

After obtaining the data, we will perform normality tests. Continuous variables will be expressed as medians with interquartile ranges or means with SD. The categorical variables will be expressed as numbers in each category or as frequencies. To explore surrogate markers of RDR, simple linear regression analysis will be performed to identify the relationships between procedure time, FT and RDR. A multiple linear regression analysis will be performed to identify the factors related to RDR. A p value of 0.05 will be considered statistically significant. All statistical analyses will be performed with JMP software (SAS Institute, Cary, North Carolina, USA).

\section{Patient and public involvement}

Clinical factors related to ERCP and interventional EUS have been retrospectively collected at two sites (Toyonaka Municipal Hospital and Kindai University). ${ }^{20} 22-24$ We used those published data to develop plans for the design or implementation of this study and to determine the research question or the outcome measures. No patients were asked to advise us on the interpretation or writing up of results. There are no plans to disseminate the results of the research to study participants, but we will consider disseminating the results of the research to the relevant patient community.

\section{Data collection}

The clinical factors have been modified to comply with local patient flow and administrative requirements and have been assessed and approved by this study steering committee. We are collecting the password-protected case report forms by email from each institution; these will be de-identified after all data have been collected, and all data queries have been addressed. A unique study identification number will identify each participant and the associated clinical data. Data collection will be performed at 3-month intervals to prevent data loss. Data analysis will take place at the central study site (Kindai University). This study does not require data monitoring due to its nature as an observational study without interventions. Data will be retained for either a minimum of 5 years after the end of this study or for 10 years after publication, whichever is later.

\section{Patient recruitment and schedule}

Patient recruitment will be carried out at the participating hospitals from May 2019 to December 2020.

In 2021, the data analysis, writing and submission of the main manuscript for publication will be carried out.

\section{Ethics and dissemination}

The results of this study will be presented at gastroenterology-, endoscopy- or radiology-related congresses and will be published in a peer-reviewed journal.

\section{DISCUSSION}

Currently, the establishment of DRLs is an international requirement for protection from medical radiation. For diagnostic radiology, national and regional DRLs are usually set at the $75 \%$ percentile of the distribution of a typical sample dose. ${ }^{25}$ All physicians or medical staff who are involved in radiological imaging or procedures under fluoroscopic guidance should be familiar with the DRL process as a tool for optimising protection. In addition, separate DRLs must be established for each country and/ or region because the equipment and procedure protocols can vary among different regions. ${ }^{25}$ However, the amount of RE depends on the procedure complexity, patient anatomy, lesion characteristics, disease severity ${ }^{11}$ and type of fluoroscopic devices ${ }^{20}$; thus, setting the upper limit of radiation use by applying uniform standards is difficult. Generally, DRLs are not dose limits and do not help distinguish between good and poor medical practices. ${ }^{25}$ Therefore, a high demand exists for a large 
amount of real-world evidence. The 2015 Japan DRLs state that the methods for establishing DRLs not only include setting radiation dose levels but also includes determining the dose quantities and units used to set the DRLs, thus standardising the methodology for dose measurements, data collection and identification of the applications of DRLs. ${ }^{12}$

Unfortunately, most gastroenterologists are unfamiliar with not only DRLs but also radiation protection because information on RE from gastrointestinal medical treatment is currently very scarce, and few RE standards, including DRLs, have been established worldwide. Given this background, the REX-GI study is planned as an observational, nationwide study in Japan. Our results will help to promote radiation optimisation and patient radiation protection in gastroenterology studies, such as digestive endoscopy, and hepatobiliary and pancreatic procedures.

\section{Publication}

After completion of this study, a main manuscript will be prepared to present the results and will be submitted to a clinical journal for peer review. This study will ensure that the public has access to the published data.

\section{Consent for publication}

The principal investigators will form a publication committee, which will include key members of this study, and the committee will grant authorship according to individual input. Investigators who do not qualify for authorship will be acknowledged by name in the final manuscript.

\section{Author affiliations}

${ }^{1}$ Department of Gastroenterology, Toyonaka Municipal Hospital, Toyonaka, Osaka, Japan

${ }^{2}$ Department of Gastroenterology and Internal Medicine, Hayashi Clinic, Suita, Japan ${ }^{3}$ Department of Gastroenterology and Hepatology, Kindai University, Osaka-Sayama, Osaka, Japan

${ }^{4}$ Department of Radiology, Kindai University, Osaka-Sayama, Osaka, Japan

${ }^{5}$ Department of Gastroenterology, The University of Tokyo, Bunkyo-ku, Tokyo, Japan

${ }^{6}$ Department of Gastroenterology, Fukui Prefectural Hospital, Fukui, Japan

${ }^{7}$ Department of Gastroenterology and Hepatology, Kansai Rosai Hospital,

Amagasaki, Hyogo, Japan

${ }^{8}$ Department of Gastroenterology, Osaka City University, Osaka, Japan

${ }^{9}$ Department of Gastroenterology, Ishikawa Prefectural Central Hospital, Kanazawa, Ishikawa, Japan

${ }^{10}$ Department of Gastroenterology, Tonan Hospital, Sapporo, Hokkaido, Japan

${ }^{11}$ Department of Gastroenterology, Cancer Institute Hospital, Japanese Foundation for Cancer Research, Tokyo, Japan

${ }^{12}$ Department of Gastroenterology and Hepatology, Suita Municipal Hospital, Suita, Osaka, Japan

${ }^{13}$ Department of Gastroenterology and Hepatology, Osaka Rosai Hospital, Sakai,

Osaka, Japan

${ }^{14}$ Department of Gastroenterology and Hepatology, Osaka General Medical Center,

Osaka, Japan

${ }^{15}$ Department of Gastroenterology, Fukushima Medical University School of Medicine, Fukushima, Japan

${ }^{16}$ Department of Gastroenterological Oncology, Hyogo Cancer Center, Akashi, Hyogo, Japan

${ }^{17}$ Department of Gastroenterology and Hepatology, Digestive Disease Center, Kitano Hospital, Osaka, Japan

${ }^{18}$ Department of Gastroenterology, Tane General Hospital, Osaka, Japan
${ }^{19}$ Department of Gastroenterology, Japanese Red Cross Medical Center, Shibuya, Tokyo, Japan

${ }^{20}$ Department of Gastroenterology, Kure Medical Center, Kure, Hiroshima, Japan

${ }^{21}$ Department of Gastroenterology and Metabolism, Nagoya City University Graduate School of Medical Sciences, Nagoya, Aichi, Japan

${ }^{22}$ Division of Gastroenterology and Hepatology, Department of Internal Medicine,

Toho University Ohashi Medical Center, Tokyo, Japan

${ }^{23}$ Department of Hepatobiliary and Pancreatic Oncology, Osaka International Cancer Institute, Osaka, Japan

${ }^{24}$ First Department of Internal Medicine, Gifu University Hospital, Gifu, Japan

Collaborators We Radiation Exposure from Gastrointestinal Fluoroscopic Procedures in Japan (REX-GI). The collaborators involved in the REX-Gl study are as follows: Mitsuhiro Fujishiro (Nagoya University Graduate School of Medicine), Masashi Yamamoto, Dai Nakamatsu, Kaori Mukai, Kei Takahashi, Aya Sugimoto, Naoto Osugi, Yu Higaki, Ryo Tomita, Tatsuya Sakamoto, Emi Meren, Kazuki Aochi, Shinji Kuriki, Li-sa Chang, and Koji Fukui (Toyonaka Municipal Hospital), Yousuke Nakai (The University of Tokyo), Takahiro Suda (Kansai Rosai Hospital), Kazuhiro Matsunaga (Ishikawa Prefectural Central Hospital), Tetsuya Sumiyoshi (Tonan Hospital), Takashi Sasaki, Atsuko Tamashiro, Hiroyuki Hatamori (Cancer Institute Hospital, Japanese Foundation for Cancer Research), Takumi Kanagawa, Yuichi Yoshida, Masafumi Naito (Suita Municipal Hospital), Shuji Ishii (Osaka General Medical Center), Takuto Hikichi (Fukushima Medical University School of Medicine), Naoki Fujimoto (Tane General Hospital), Ikuya Miki (Hyogo Cancer Center), Yuzuru Tamaru (National Hospital Organization Kure Medical Center and Chugoku Cancer Center), Hiromi Kataoka, Kazuki Hayashi (Nagoya City University Graduate School of Medical Sciences), and Hiroaki Shigoka (Toho University Ohashi Medical Center).

Contributors TN, SH (Toyonaka Municipal Hospital) and MT (Kindai University) designed this study. MH (Kindai University) critically reviewed the protocol. TN, SH (Toyonaka Municipal Hospital), MT (Kindai University), HK (the University of Tokyo), KH (Fukui Prefectural Hospital), SY (Kansai Rosai Hospital), HM (Osaka City University), HD (Ishikawa Prefectural Central Hospita), HI (Tonan Hospital), TYoshio (Cancer Institute Hospital), KN (Suita Municipal Hospital), TYamada (Osaka Rosai Hospital), TYakushijin (Osaka General Medical Center), TT (Fukushima Medical University School of Medicine), HT (Hyogo Cancer Center), AK (Kitano Hospital), SA (Tane General Hospital), YI (Japanese Red Cross Medical Center), TK (National Hospital Organization, Kure Medical Center and Chugoku Cancer Center), YH (Nagoya City University Graduate School of Medical Sciences), IM (Toho University Ohashi Medical Center), KI (Osaka International Cancer Institute), TI (Gifu University Hospital), KM and MI (Toyonaka Municipal Hospital) participated in this study and recruited the patients.

Funding This research received clinical research grants from the Japanese Society of Gastroenterology.

Map disclaimer The depiction of boundaries on the map in this article does not imply the expression of any opinion whatsoever on the part of BMJ (or any member of its group) concerning the legal status of any country, territory, jurisdiction or area or of its authorities. The map is provided without any warranty of any kind, either express or implied.

Competing interests None declared.

Patient consent for publication Not required.

Ethics approval This observational study will be conducted in accordance with the principles of the Declaration of Helsinki, and approval has been obtained from the Institutional Review Board of Toyonaka Municipal Hospital (25 April 2019) and the institutional review board of each participating facility. The need for informed consent will be waived via the opt-out method on each hospital website.

Provenance and peer review Not commissioned; externally peer reviewed.

Open access This is an open access article distributed in accordance with the Creative Commons Attribution Non Commercial (CC BY-NC 4.0) license, which permits others to distribute, remix, adapt, build upon this work noncommercially, and license their derivative works on different terms, provided the original work is properly cited, appropriate credit is given, any changes made indicated, and the use is non-commercial. See: http://creativecommons.org/ licenses/by-nc/4.0/.

ORCID iD

Tsutomu Nishida http://orcid.org/0000-0003-4037-9003 


\section{REFERENCES}

1 Henry MF, Maender JL, Shen Y, et al. Fluoroscopy-induced chronic radiation dermatitis: a report of three cases. Dermatol Online $J$ 2009;15:3.

2 NCRP. Commentary No. 27: implications of recent epidemiologic studies for the Linear-Nonthreshold model and radiation protection 2018. Available: https://ncrponline.org/wp-content/themes/ncrp/ Pub_announcements/Commentary_No27_overview.pdf [Accessed 9 Jul 2019].

3 Park TH, Eichling JO, Schechtman KB, et al. Risk of radiation induced skin injuries from arrhythmia ablation procedures. Pacing Clin Electro 1996;19:1363-9.

4 de González AB, Darby S. Risk of cancer from diagnostic Xrays: estimates for the UK and 14 other countries. The Lancet 2004;363:345-51.

5 Jaffe D, Bowden GT. lonizing radiation as an initiator: effects of proliferation and promotion time on tumor incidence in mice. Cancer Res 1987;47:6692-6.

6 Mathews JD, Forsythe AV, Brady Z, et al. Cancer risk in 680,000 people exposed to computed tomography scans in childhood or adolescence: data linkage study of 11 million Australians. BMJ 2013;346:f2360.

7 Khoramian D, Sistani S, Hejazi P. Establishment of diagnostic reference levels arising from common CT examinations in Semnan County, Iran. Polish Journal of Medical Physics and Engineering 2019;25:51-5.

8 Radiological protection and safety in medicine. A report of the International Commission on radiological protection. Ann ICRP 1996;26:1-47.

9 The 2007 recommendations of the International Commission on radiological protection. ICRP publication 103. Ann ICRP 2007;37:1-332.

10 ICRP publication 105. radiation protection in medicine. Ann ICRP 2007;37:1-63.

11 Vañó E, Miller DL, Martin CJ, et al. ICRP publication 135: diagnostic reference levels in medical imaging. Ann ICRP 2017;46:1-144.

12 Dignostic reference levels based on latest surveys in Japan -Japan DRLs 2015, 2015. Available: http://www.radher.jp/J-RIME/report/ DRLhoukokusyoEng.pdf [Accessed 9 Jul 2019].
13 Hart D, Wall BF. Uk population dose from medical X-ray examinations. Eur J Radiol 2004;50:285-91.

14 Dumonceau J-M, Garcia-Fernandez F, Verdun F, et al. Radiation protection in digestive endoscopy: European Society of digestive endoscopy (ESGE) guideline. Endoscopy 2012;44:408-24.

15 Hayashi S, Takenaka M, Hosono M, et al. Radiation exposure during image-guided endoscopic procedures: the next quality indicator for endoscopic retrograde cholangiopancreatography. WJCC 2018;6:1087-93.

16 Adler DG, Lieb JG, Cohen J, et al. Quality indicators for ERCP. Gastrointest Endosc 2015;81:54-66.

17 Matsuda K, Tanaka K, Fujishiro M, et al. Design paper: Japan Endoscopy Database (JED): A prospective, large database project related to gastroenterological endoscopy in Japan. Digestive Endoscopy 2018;30:5-19.

18 Diagnostic reference levels in medical imaging: review and additiona advice. Ann ICRP 2001;31:33-52.

19 Lynskey GE, Powell DK, Dixon RG, et al. Radiation protection in interventional radiology: survey results of attitudes and use. Journal of Vascular and Interventional Radiology 2013;24:1547-51.

20 Hayashi S, Nishida T, Matsubara T, et al. Radiation exposure dose and influencing factors during endoscopic retrograde cholangiopancreatography. PLoS One 2018;13:e0207539.

21 Bf S. Bekanntmachung Der aktualisierten diagnostischen Referenzwerte für diagnostische und interventionelle Röntgenanwendungen Vom 22, 2016. Available: https://www. klinikum.uni-heidelberg.de/fileadmin/strahlenschutz/linkimages/PDF/ DRW-XRay-2016-07-15.pdf [Accessed 9 Nov 2019].

22 Hayashi S, Nishida T, Shimakoshi H, et al. Mo2020 novel processing engine for X-ray fluoroscopic images (Faice-V NS1) can reduce radiation exposure in the procedure of ERCP but keep the quality of images. Gastrointest Endosc 2017;85:AB518.

23 Hayashi S, Higaki Y, Tomita R, et al. 940 disease site and processing engine affect radiation exposure dose during ERCP. Gastrointest Endosc 2018;87:AB137.

24 Takenaka M, Hayashi S, Nishida T, et al. Mo1090 examination of actual radiation exposure dose of the patients who performed EUSGUIDED drainage (EUS-BD/EUS-PD/EUS-CD). Gastrointest Endosc 2019;89:AB444-5

25 Vassileva J, Rehani M. Diagnostic reference levels. AJR Am J Roentgenol 2015;204:W1-3. 